\title{
Is Prevention Focus less effective for Performance?
}

\author{
Miki Toyama', Masato Nagamine ${ }^{1}$, Li Tang $^{1}$, Shuhei Miwa², Atsushi Aikawa1 \\ ${ }^{1}$ University of Tsukuba, Japan / Center for Research on Educational Testing \\ ${ }^{2}$ Kansai Gaidai University, Japan / Center for Research on Educational Testing
}

\begin{abstract}
Regulatory focus theory (Higgins, 1997) proposes that individuals tend to adopt one of two fundamental motives for guiding their goal pursuit: the motive for growth and advancement (promotion focus) or the motive for safety and security (prevention focus). Moreover, the regulatory focus is known to affect performance. We examined the effect of regulatory focus on the performance of a task succeeding a preceding task. University student participants $(N=62)$ were induced to have a promotion- or a prevention-focused orientation and requested to conduct a more or a less important task, which was followed by a more important task. Results indicated that when the prevention-focused orientation was activated, participants did not spend much effort on achieving the less important task, and performance decreased compared to when the promotion-focused orientation was activated. This result could be explained by the increased intention for conserving cognitive resources because the participants knew that engaging in the task is not important. On the other hand, the participants demonstrated higher performance for the more important task that was implemented later, compared to when the promotion-focused orientation was activated. The above results suggest that cognitive resources are allocated intentionally in prevention-focused conditions.
\end{abstract}

Keywords: performance; prevention focus; promotion focus; regulatory focus 